Category: Mitochondrial Diseases

\title{
Development of Cerebral Organoid cultures for the study of the neuronal pathomolecular mechanisms of Mitochondrial NeurogastroIntestinal Encephalomyopathy (MNGIE)
}

Dario Pacitti ${ }^{1}$ (p1406231@sgul.ac.uk), Niranjanan Nirmalananthan², Bridget E. Bax ${ }^{1}$

${ }^{1}$ St George's University of London, Molecular and Cell Sciences Institute, Cell Biology and Genetics Research Centre

2 St Georges University Hospitals NHS Foundation Trust

\section{Background:}

Mitochondrial Neurogastrointestinal Encephalomyopathy (MNGIE) is a rare metabolic disorder caused by a mutation in the thymidine phosphorylase (TP) gene, leading to secondary aberrations to the mitochondrial genome. The disease is clinically characterised by neurological and gastrointestinal dysfunctions, including neuropathy and leukoencephalopathy.

Aims:

The understanding of molecular mechanisms of MNGIE affecting the nervous system is hindered by the lack of a representative model. We are developing in vitro 3-D cerebral organoids as a neuronal model for MNGIE.

Methods:

Patient-derived somatic cells were reprogrammed to generate bona fide induced pluripotent stem cell (iPSC) lines. Pluripotent cells were differentiated into neuroepithelium, embedded in Matrigel and cultured in retinoic acid within bioreactors to generate CNS organoids. TP activity of organoids was monitored by weekly UPLC analysis of spent culture medium and intact organoids were sampled for morphological evaluations and for RNA extraction.

Results:

The iPSCs were positive to the immunofluorescence detection of pluripotency markers TRA-1-60 and SOX2. Germ layer differentiation assay indicated that iPSCs were capable of differentiating into Endoderm, Mesoderm and Ectoderm tissues. Karyotyping indicated no chromosomal abnormalities in generated lines. iPSCs successfully generated cerebral organoids displaying presence of neuromelanin pigments and cystic structures. Levels of thymidine quantified by UPLC, showed no significant changes in concentration for MNGIE whereas in Control organoids, levels of thymidine decreased over weeks, indicating that thymidine phosphorylase is active.

\section{Conclusion:}

We have established a neuronal organoid model of MNGIE which will be employed in whole genome expression profiling to investigate differential expression between MNGIE and Control and gene editing for the generation of a rescue phenotype. 